\title{
Mechanisms of Caspase-Independent Neuronal Death: Energy Depletion and Free Radical Generation
}

\author{
Isabelle C. J. Lang-Rollin, ${ }^{1}$ Hardy J. Rideout, ${ }^{1}$ Manish Noticewala, ${ }^{1}$ and Leonidas Stefanis ${ }^{1,2}$ \\ Departments of ${ }^{1}$ Neurology and ${ }^{2}$ Pathology, Columbia University, New York, New York 10032
}

Cultured rat embryonic cortical neurons undergo apoptosis when treated with the topoisomerase-I inhibitor camptothecin. Pharmacological or molecular caspase inhibition prevents apoptosis, but the neurons still die in a delayed nonapoptotic manner. Here we examine the mechanisms leading to such caspase-independent death, focusing on events related to mitochondrial malfunction, which accompanies this delayed death. Given that mitochondria are the major source of ATP in primary neurons, we examined the cellular energy state. Mitochondrially generated ATP was specifically reduced in neurons treated with camptothecin and Boc-aspartyl-fluoromethylketone. Augmentation of cellular ATP by manipulation of the glucose content in the cultures led to an increase in survival specifically in delayed caspase-independent but not early caspase-dependent death. As another possible consequence of mitochondrial malfunction, we found an induction of reactive oxygen species in delayed death. The free radical scavenger Tempol, but not other classes of antioxidants, reduced oxidative stress and promoted survival. Other potential events known to be a direct or indirect consequence of mitochondrial dysfunction, such as the induction of autophagy, release of apoptosis-inducing factor, or opening of the mitochondrial permeability transition pore, were not found to play a significant role in caspase-independent neuronal death. Combining the strategies of increasing intracellular ATP and reducing free radicals led to an additive increase in neuronal survival. We conclude that energy failure and free radical generation contribute to caspase-independent neuronal death. Both could represent potential targets for therapeutic interventions complementary to caspase inhibition.

Key words: ATP; caspase inhibition; oxidative stress; autophagy; AIF; mitochondria; cytochrome $c$

\section{Introduction}

Inhibition of caspases has been suggested as a therapeutic strategy in neurodegenerative and acute neurological disorders (Schulz et al., 1999; Rideout and Stefanis, 2001). Indeed, in some animal models of disease, pharmacological caspase inhibitors have been shown to offer some protection against neuronal death and, in some cases, to ameliorate clinical manifestations (Cutillas et al., 1999; Li et al., 2000; Cao et al., 2002; Knoblach et al., 2002). However, there is increasing evidence, primarily from neuronal cell culture models, that caspase inhibition offers only transient protection, and that caspase-independent death eventually occurs (Stefanis et al., 1999; Keramaris et al., 2000; Selznik et al., 2000). Elucidating the mechanisms of caspase-independent death cannot only provide fundamental insights into biologically relevant cell death pathways but also identify targets for therapeutic intervention that are complementary to caspase inhibition.

Received Sept. 2, 2003; revised Sept. 29, 2003; accepted 0ct. 1, 2003.

This work was supported by the American Parkinson's Disease, The Matheson, and the Parkinson's Disease Foundations, a Burroughs Wellcome Career Award in Biomedical Sciences (L.S.), and the Deutsche Forschungsgemeinschaft (I.L.-R.). We thank Drs. Harry Ischiropoulos, Pat Sullivan, Eric Schon, and Serge Przedborski for helpful discussions, Drs. Kroemer and Susin for their generous gift of an apoptosis inducing factor antibody, Dimitris Thanos for the use of the luminometer, and Mary Schoenebeck for her invaluable technical assistance with the electron microscopy studies.

Correspondence should be addressed to Leonidas Stefanis, Assistant Professor of Neurology and Pathology, Department of Neurology, Columbia University, 650 West 168th Street, Black Building, Room 326, New York, NY 10032. E-mail: Is76@columbia.edu.

Copyright $\odot 2003$ Society for Neuroscience 0270-6474/03/2311015-11\$15.00/0
In this study, we examined the mechanism of caspaseindependent death of rat embryonic cortical neurons treated with camptothecin (cam) and the general caspase inhibitor Bocaspartyl-fluoromethylketone (BAF). The topoisomerase-I inhibitor camptothecin induces rapid apoptotic death in these cultures. Studies of the mechanism of camptothecin-induced neuronal apoptosis suggest a model in which DNA damage leads to parallel activation of cell cycle components and p53. This leads to Bax-dependent release of cytochrome $c$ from the mitochondria and consequent activation of caspases (Xiang et al., 1998; Stefanis et al., 1999; Morris et al., 2001). We have shown previously that the general pharmacological caspase inhibitor BAF inhibits apoptotic death in this model; however, neurons subsequently undergo a delayed caspase-independent cell death lacking the nuclear features of apoptosis (Stefanis et al., 1999). BAF does not prevent loss of cytochrome $c$ and only delays loss of mitochondrial transmembrane potential (MTP) in this model. However, Bax-deficient neurons, which do not undergo mitochondrial alterations, almost completely resist camptothecin-induced death (Xiang et al., 1998; Morris et al., 2001). Thus, the mitochondria lie at a crucial checkpoint in the course of events leading to cell death. If mitochondrial function is disrupted, even complete blockade of the apoptotic pathway downstream of this disruption does not lead to sustained survival. Mitochondrial dysfunction can lead to a disruption of electron transport and energy metabolism, as well as an impaired regulation of the cellular redox potential (Green and Reed, 1998). Mitochondrial alterations 
have also been linked to autophagy (Lemasters et al., 1998; Xue et al., 1999, 2001; Elmore et al., 2001). In addition, mitochondria have been shown to release, in addition to cytochrome $c$, other death-inducing proteins such as the apoptosis-inducing factor (AIF), which may act in a caspase-independent manner (Daugas et al., 2000).

In the current study, we examined the role of several potential events known to be a direct or indirect consequence of mitochondrial dysfunction in caspase-independent neuronal death. We focus in particular on the generation of reactive oxygen species (ROS), induction of autophagy, release of AIF, and mitochondrial energy production.

\section{Materials and Methods}

\section{Cell culture}

Primary cortical neurons from embryonic day (E) 18 rats were prepared as described previously (Friedman, 1993; Stefanis et al., 1999; Rideout et al., 2001; Rideout and Stefanis, 2002). Briefly, after dissection of the cortical mantle, the tissue was dissociated by mechanical trituration. The cells were then resuspended in neurobasal medium supplemented with B-27, 0.5 mM L-glutamine, and 1\% penicillin-streptomycin (Invitrogen, Grand Island, NY) and plated in poly-D-lysine-coated tissue cultures dishes. Using such procedures, free of the use of serum, $>98 \%$ of the cultured cells represent neurons (Friedman et al., 1993; Rideout and Stefanis, 2002). For certain experiments, cells were resuspended instead in other specific media, either neurobasal medium supplemented with antioxidant-free B-27, or glucose-free RPMI supplemented with regular $\mathrm{B}-27$ (Invitrogen) and $5 \mathrm{~mm}$ pyruvate. Cultures were maintained at $37^{\circ} \mathrm{C}$ in a humidified atmosphere containing $5 \% \mathrm{CO}_{2}$.

\section{Application of reagents}

On the first or second day after plating, cortical neurons were treated with $10 \mu \mathrm{M}$ cam alone or in combination with $100 \mu \mathrm{M}$ Boc-aspartyl (OMe)-fluoromethylketone (Enzyme Systems Products, Livermore, CA) as described previously (Stefanis et al., 1999). In some experiments, the neurons were treated concomitantly with one of the following substances: vitamin E (0.1-1 mg/ml), Mn (III)tetrakis(4-benzoic acid) porphyrin chloride (MnTBAP; $100 \mu \mathrm{M}$; Calbiochem, San Diego, CA), Mn(III) tetrakis (1-methyl-4-pyridyl) porphyrin pentachloride (MnTMPyP; $100 \mu \mathrm{m}$; Calbiochem), Trolox (100-200 $\mu \mathrm{g} / \mathrm{ml}$; Calbiochem), $\mathrm{N}$-acetylcysteine (NAC) (0.1-1 mM), L- $\gamma$-glutamyl-L-cysteinyl ethyl glycinate (GSH-MEE; $500 \mu \mathrm{M}$, Calbiochem), 4,5, dihydroxybenzenedisulfonic acid (Tiron; $5 \mathrm{mM}$ ), cyclosporine A (1 $\mu \mathrm{M})$, bonkrekic acid (5-50 $\mu \mathrm{M})$, 3-methyladenine (10 mM), bafilomycin (2.5 nM), 4-hydroxytetramethyl-piperidine-1-oxyl (Tempol; 5 mm; Calbiochem), or D-glucose $(4 \mathrm{mg} / \mathrm{ml})$. If not otherwise specified, reagents were purchased from Sigma (St. Louis, MO).

\section{Assessment of survival}

Twelve or twenty hours after application of the above reagents, cortical neurons were lysed, and the number of intact nuclei was counted in a hemacytometer as described previously (Rukenstein et al., 1991; Stefanis et al., 1999). This method has been shown to be reproducible and accurate and to correlate well with other methods of assessing cell survivaldeath (Stefanis et al., 1997, 1999). Positive results were verified by counting the number of viable (calcein AM positive; see below) neurons in a defined number of fields in each well. All counts were conducted in a blinded manner. Cell counts were performed in triplicate and are reported as means \pm SEM. The data are expressed as the percentage of the number of neurons in the control cultures (i.e., cultures that were treated with no additives) at each time point. At least 100 neurons per well were counted for each one in control conditions. All data shown are representative of at least three replicate experiments.

\section{Application of fluorescent dyes}

Lysosomal function. E18 cortical neuron cultures were treated with various agents as described above and then labeled with LysoTracker Red, a fluorescent dye that accumulates specifically in acidic organelles. Thus, LysoTracker Red primarily labels functional lysosomes, which are the intracellular organelles that contain acidic enzymes and have a low $\mathrm{pH}$ (Stefanis et al., 2001). The cultures were incubated at $37^{\circ} \mathrm{C}$ for $20 \mathrm{~min}$ with $50 \mathrm{~nm}$ Lysotracker Red and then, after one rinse and replacement of the medium, they were visualized by fluorescence microscopy, and representative photomicrographs were obtained.

ROS generation. Two separate dyes that react with reactive oxygen species and thus lead to the emission of a fluorescent signal were used. In these experiments, to assess only cells that remained alive but yet had accumulated ROS, we prelabeled the cultures with the viability indicator calcein AM (4 $\mu \mathrm{M})$. To emit a fluorescent signal at the fluorescein range, this dye needs to be hydrolyzed by intracellular esterases and prevented from diffusing from the cell by an intact outer cell membrane. The cultures were incubated in the presence of calcein $\mathrm{AM}$ for $30 \mathrm{~min}$ at $37^{\circ} \mathrm{C}$ and then rinsed and incubated for another $30 \mathrm{~min}$ in the absence of calcein AM to allow its diffusion from neurons with a damaged cell membrane. Subsequently, the cultures were exposed to medium containing dihydroethidium (DHE; $1 \mu \mathrm{M}$ ) for $30 \mathrm{~min}$ at $37^{\circ} \mathrm{C}$. When DHE reacts with intracellular ROS, it fluoresces in the rhodamine spectrum within cell nuclei (Krohn et al., 1998). After DHE labeling, the cultures were rinsed once, replenished with fresh medium, and visualized under a fluorescence microscope. The percentage of green-fluorescing (i.e., viable) cells that showed red nuclear fluorescence (i.e., ROS generation) was assessed in each setting. Counts were performed in triplicate and are reported as means \pm SEM. As a complementary approach, we also used the dye dichlorodihydrofluorescein diacetate (DCF; $75 \mu \mathrm{M}$ ), which also fluoresces within cells after exposure to ROS (Hsu et al., 2000).

Mitochondrial transmembrane potential. Cortical neuron cultures after various treatments, as described above, were incubated with medium containing tetramethylrhodamine methyl ester (TMRM; $50 \mathrm{nM}$ ) and calcein $\mathrm{AM}(4 \mu \mathrm{M})$ for $20 \mathrm{~min}$ at $37^{\circ} \mathrm{C}$. After a rinse, and an additional 30 min incubation of the cultures in fresh medium, we assessed the percentage of live (calcein-positive) neurons that still maintained TMRM fluorescence. Counts were conducted in a blinded manner. Cell counts were performed in triplicate and are reported as means \pm SEM. TMRM fluorescence has been validated across other methods as an accurate way to assess MTP (Metivier et al., 1998; Rideout et al., 2001). All fluorescent dyes were obtained from Molecular Probes (Eugene, OR).

\section{Immunofluorescence}

Cortical neuron cultures on glass slides were first incubated for $30 \mathrm{~min}$ at $37^{\circ} \mathrm{C}$ with the mitochondrial dye Mitotracker Green (500 nM). This dye labels mitochondria regardless of transmembrane potential and thus serves as a mitochondrial marker. The cultures were then rinsed three times with PBS and fixed with $3.7 \%$ paraformaldehyde and $0.19 \%$ picric acid in PBS for $20 \mathrm{~min}$ at $4^{\circ} \mathrm{C}$. After rinsing with PBS, the cells were incubated at room temperature (RT) for 30 min with blocking solution [ $10 \%$ normal goat serum (NGS) in PBS with $0.4 \%$ Triton X-100]. They were then incubated for $1 \mathrm{hr}$ at RT with a mouse monoclonal antibody against AIF (Santa Cruz Biotechnology, Santa Cruz, CA) diluted 1:100 in $1 \%$ NGS in PBS with $0.4 \%$ Triton X-100. After three washes with PBS, the secondary antibody (Jackson ImmunoResearch, West Grove, PA; 1:100 of goat Cy3-conjugated anti-mouse, in 1\% NGS in PBS with $0.4 \%$ Triton $\mathrm{X}-100$ ) was applied for $30 \mathrm{~min}$ at RT. After additional washes, the nuclear dye TOTO-3 iodide (Molecular Probes; 1:1400) was applied to the cells and then rinsed with PBS. Coverslips were applied, and the cells were then visualized under a confocal microscope.

As an alternative for AIF immunostaining, we used similar procedures as described above to immunostain cultures with a combination of a rabbit anti-AIF antiserum (1:500; generously provided by Drs. Kroemer and Susin, Centre National de la Recherche Scientifique, Institut Gustav Roussy, Villejuif, France) and a monoclonal antibody directed against subunit IV of cytochrome oxidase (COX; 1:2500; Molecular Probes). COX forms part of the mitochondrial matrix and is thus not released during events leading to the release of other mitochondrial factors from the intermembrane space.

\section{Subcellular fractionation and Western immunoblotting}

To identify changes in the subcellular localization of proteins, enriched mitochondrial and nuclear fractions were obtained using differential 
centrifugation. Briefly, cells were rinsed in PBS and then triturated from the dish and centrifuged. The pellets were resuspended in extraction buffer A (300 mu sucrose, 1 mm EDTA, 20 mm morpholinopropanesulfate) and protease inhibitors (Roche Products, Indianapolis, IN). After 30 min on ice, cells were lysed with 30 strokes of a glass Dounce homogenizer. The nuclei and any unbroken cells were then centrifuged at $100 \times$ $g$ for $10 \mathrm{~min}$ at $4^{\circ} \mathrm{C}$. The supernatant was centrifuged at $16,000 \times g$ for 20 $\min$ at $4^{\circ} \mathrm{C}$ to obtain an enriched mitochondrial heavy membrane fraction. Crude nuclear fractions and unbroken cells were meanwhile resuspended in lysis buffer B, which lyses outer cell but not nuclear membranes. This is the same buffer we used to lyse cells for nuclear counting (Rukenstein et al., 1991). The nuclei were then washed three times by centrifugation and resuspension in PBS. The enriched mitochondrial and nuclear pellets were then lysed in buffer C (25 mM HEPES, pH 7.4, 5 mm EDTA, 1 mM EGTA, 5 $\mathrm{mm} \mathrm{MgCl}$, and protease inhibitors) containing $1 \%$ Triton X-100 and submitted to Western immunoblotting. Protein concentrations were measured using the Bradford reagent (Bio-Rad, Hercules, CA). Equal volumes of $2 \times$ sample buffer were added to the lysates. The samples were then boiled for 10 min and subsequently resolved by electrophoresis on SDS-PAGE, transferred to nitrocellulose membranes, blocked in 5\% nonfat milk in PBS, and incubated overnight at $4^{\circ} \mathrm{C}$ in $3 \%$ bovine serum albumin in PBS with various primary antibodies: mouse anti-AIF (1:500; Santa Cruz Biotechnology), mouse anti-cytochrome $c$ (1:1000; PharMingen, San Diego, CA), mouse anti-subunit IV of COX (1:2500; Molecular Probes), used as a mitochondrial marker, and mouse anti-retinoblastoma protein (pRb) (1:1000; PharMingen), used as a nuclear marker. The blots were then washed in PBS with $0.2 \%$ Tween 20, incubated for $1 \mathrm{hr}$ at RT with goat anti-mouse IgG antibody (Pierce, Rockford IL) at 1:10,000 in 5\% milk in PBS, washed again, and then processed with the Pierce supersignal substrate system, according to the instructions of the manufacturer.

\section{Transfection of cortical neurons}

Cortical neurons were transfected with a vector [pCMS-enhanced green fluorescent protein (EGFP); Clontech, Cambridge, UK] encoding for EGFP alone or in addition to EGFP for human Superoxide dismutase 1 (SOD1) using a modified Lipofectamine 2000 reagent protocol (Life Sciences, Hialeah, FL). The correlation between expression of EGFP and SOD was $\sim 90 \%$ as evaluated by immunocytochemistry using an antiSOD antibody (Sigma). Neurons were then treated as above, and survival was assessed by counting the number of green fluorescent neurons in a defined area of the well at the time of treatment and after $20 \mathrm{hr}$. ROS generation in green fluorescing neurons was assessed in parallel by DHE staining as above.

\section{Electron microscopy}

Cultures were treated as above and then fixed for $1 \mathrm{hr}$ at $4^{\circ} \mathrm{C}$ in Sorensen's buffer (0.1 M phosphate buffer; 1:4 monobasic:dibasic) containing $2.5 \%$ glutaraldehyde. Cells were then scraped off and centrifuged at $3000 \times g$ for $5 \mathrm{~min}$. The pellet was maintained in Sorensen's buffer without glutaraldehyde and then processed for electron microscopy (EM) using standard methods (Tennyson et al., 1993; Stefanis et al., 2001).

\section{Measurement of ATP levels}

Neurons plated in $35 \mathrm{~mm}$ dishes or in 12 -well dishes were rinsed from the dish in a small volume of PBS. A $50 \mu$ l aliquot was submitted to the ATP luminometric assay kit (Roche Products) following the instructions of the manufacturer. The remaining neurons were lysed, and the intact nuclei were counted in a blinded manner as described above. ATP levels were then normalized to cell numbers. Measurements were performed in triplicate and are reported as mean \pm SEM. To measure specifically the ATP cellular content that was not derived from the mitochondria, some neurons were pretreated for $15 \mathrm{~min}$ with the mitochondrial ATP synthase inhibitor oligomycin, which blocks ATP production through the respiratory chain $(2 \mu \mathrm{g} / \mathrm{ml}$; Sigma).

\section{Results}

The autophagic-lysosomal pathway is activated in caspase-independent cell death, but its inhibition does not offer protection

We and others have shown that the DNA damaging agent camptothecin leads to caspase-dependent apoptosis of rodent embryonic cortical neurons (Morris and Geller, 1996; Park et al., 1998; Xiang et al., 1998; Stefanis et al., 1999; Keramaris et al., 2000; Morris et al., 2001; Rideout et al., 2001). Pharmacological or molecular inhibition of caspases prevents apoptosis in this model but leads to a delayed death lacking some of the classical features of apoptosis, such as chromatin fragmentation or terminal deoxynucleotidyl transferase-mediated biotinylated UTP nick end labeling staining (Stefanis et al., 1999; Keramaris et al., 2000). It has been postulated that mitochondrial alterations might play a key role in this delayed cell death (Stefanis et al., 1999; Keramaris et al., 2000; Xue et al., 2001; Chang and Johnson, 2002; Cregan et al., 2002). In certain situations, mitochondrial dysfunction may lead to activation of autophagy (Lemasters et al., 1998; Elmore et al., 2001). Autophagy is the process through which double membrane structures, thought to emanate from the endoplasmic reticulum, engulf intracellular organelles or parts of the cytoplasm. Such autophagic bodies are converted to autophagolysosomes and eventually mature lysosomes through fusion with lysosomes. The contents of autophagic bodies are thus eventually digested by lysosomal acidic enzymes (Larsen and Sulzer, 2002).

To study whether the process of autophagy is activated in caspase-independent death in our model, we treated E18 rat cortical neurons with camptothecin and the general caspase inhibitor BAF for $20 \mathrm{hr}$ and submitted the cultures to ultrastructural analysis, which is the gold standard for the identification of this process (Xue et al., 1999; Larsen and Sulzer, 2002). At this time point, as we have shown previously, caspase-independent cell death occurs in this model (Stefanis et al., 1999). In parallel, we examined cultures treated with camptothecin alone for $10 \mathrm{hr}$. As expected, clear features of apoptosis were observed in this early caspase-dependent death (Fig. $1 B$ ). In contrast, although some chromatin condensation and margination was observed at late stages in the delayed caspase-independent cell death (Fig. 1C,D), nuclear features like the intense chromatin clumping and fragmentation of the nucleus as seen in classical apoptotic cell death were not found. Many cells in these cultures treated with the combination of camptothecin and BAF showed clear features of autophagy (Fig. 1C,D). Counts of autophagic bodies defined as intracytoplasmic double-membrane structures engulfing cellular material were performed blindly in random EM images and confirmed an activation of autophagy in delayed death (Fig. $2 \mathrm{~A}$ ).

Autophagy has been invoked as a mechanism through which neonatal rat sympathetic neurons undergo caspase-independent cell death (Xue et al., 1999). We therefore tested whether the autophagy inhibitor 3 methyladenine (3-MA) (Seglen and Gordon, 1982; Xue et al., 1999; Petiot et al., 2000) would attenuate caspase-independent death in our model. We treated the cultures with camptothecin and BAF and added 3-MA $8 \mathrm{hr}$ later. This was done because 3-MA partially inhibited caspase-dependent cell death (data not shown), and we did not wish this effect to interfere with the later caspase-independent death. As shown in Figures $1, E$ and $F$, and $2 B, 3$-MA partially inhibited the activation of autophagy in neurons treated with camptothecin and BAF. However, caspase-independent death was not attenuated (Fig. 2C).

To assess whether the induction of autophagy was associated with a general activation of the lysosomal pathway, we used the 


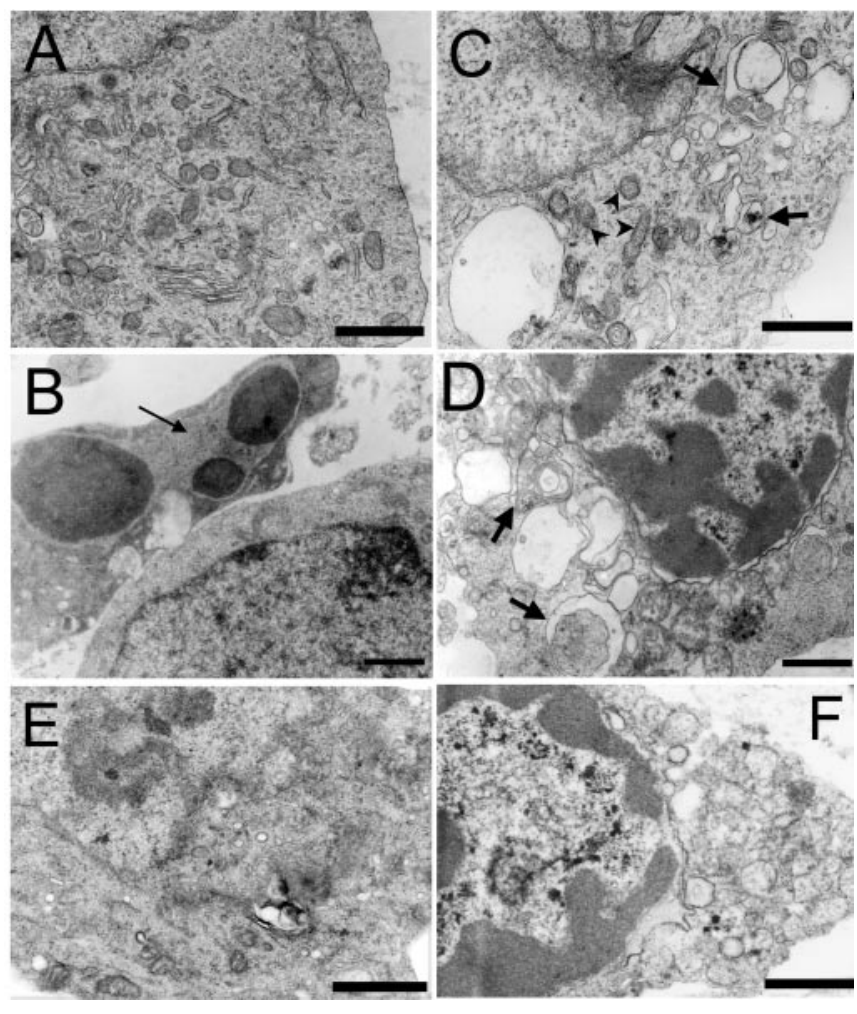

Figure 1. Activation of autophagy in caspase-independent cell death. $A-F$, EM was performed in E18 cortical neurons cultured for $2 \mathrm{~d}$ with no additives $(A)$, treated with $10 \mu \mathrm{m}$ camptothecin alone for $10 \mathrm{hr}(B)$, or $10 \mu \mathrm{m}$ camptothecin plus $100 \mu \mathrm{m} B A F$ for $20 \mathrm{hr}(C, D)$ with or without the addition of 3-MA with an $8 \mathrm{hr}$ delay $(E, F) . B$, A classical apoptotic cell with condensed and fragmented chromatin within the nucleus (arrow) and a shrunken cell body can be appreciated. We found no such cells after the combined application of camptothecin and BAF. Nuclear alterations in this delayed death do not progress beyond some degree of chromatin condensation and margination as seen in $D$. Instead, many cells showed signs of autophagy $(C, D)$. The arrows point to autophagolysosomes. Such marked autophagic features were not seen in control or camptothecin alone-treated cultures. $E$, $F$, Features of autophagy were decreased by 3-MA $(E, F)$, even in clearly degenerating neurons $(F)$. Although some autophagolysosomes appear to engulf mitochondria (C, top arrow), normal looking mitochondria can still be found in the cytoplasm ( $C$, arrowheads). Scale bar, $1 \mu \mathrm{m}$.

dye Lysotracker Red. We observed intense, globular Lysotracker Red labeling in delayed death. This was attenuated in the presence of 3-MA (data not shown), suggesting that the increased autophagy led to a general activation of the lysosomal pathway.

We also used an alternative strategy to inhibit the pathway of lysosomal activation by applying the pharmacological agent bafilomycin, which is a specific inhibitor of the vacuolar ATPase pump that is responsible for the acidification of lysosomes (Bowman et al., 1988). Bafilomycin blocks the fusion of autophagic bodies with lysosomes and would thus be expected to lead to an increase of ultrastructural evidence of autophagy but a functional inhibition of the lysosomal pathway (van Deurs et al., 1996; Yamamoto et al., 1998). Bafilomycin is usually used at concentrations of 50-100 nM. However, we found that at these concentrations it was markedly toxic to our cultures. We therefore applied this agent at a concentration of $2.5 \mathrm{~nm}$, which still completely inhibited the acidification of lysosomes, as judged by complete loss of specific Lysotracker Red labeling. Bafilomycin led to an increase in ultrastructural features of autophagy and was not toxic over the period of $24 \mathrm{hr}$ (data not shown). Despite this, it also failed to protect against caspase-independent death (Fig. 2C).
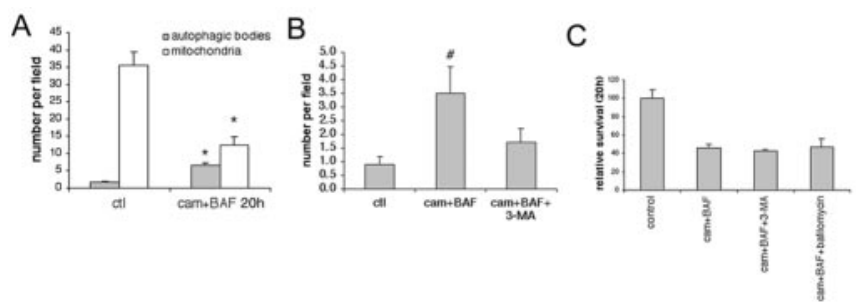

Figure 2. $\quad A$, Increase in autophagic bodies in caspase-independent neuronal death. Random electron microscopic images of cortical neurons treated with no additive (ctrl) or with camptothecin plus BAF (cam + BAF) for $20 \mathrm{hr}$ were analyzed. Autophagic bodies defined as intracytoplasmic double-membrane bound structures engulfing cellular material were counted in a blinded manner. In parallel, mitochondria were counted in both conditions. Numbers are reported as the mean \pm SEM ( $n=20 ;{ }^{*} p<0.01$; Student's $t$ test). $B$, Addition of 3-MA inhibits the activation of autophagy. The addition of $3-\mathrm{MA}(10 \mathrm{~mm}$; added with an $8 \mathrm{hr}$ delay) to cortical neurons treated with camptothecin and BAF reduces the amount of autophagic bodies, counted as in $A$ in a blinded manner in random electron microscopic images. Numbers are reported as the mean \pm SEM $(n=10)$. Although, as in $A$, there was an increase in the number of autophagic bodies in cam + BAF-treated cultures $(p=0.027)$, cultures treated with the addition of 3-MA showed no such increase compared with controls $(p=0.21)$, implying that autophagy was not activated in the presence of 3-MA.C, Inhibitors of autophagy or of the vacuolar proton pump do not protect against caspase-independent cell death. Cortical E18 neurons were treated with camptothecin plus BAF in the presence or absence of the autophagy inhibitor 3-MA (10 mM) or of the inhibitor of the vacuolar ATPase proton pump bafilomycin $(2.5 \mathrm{~nm})$. After $20 \mathrm{hr}$, the number of surviving neurons was assessed by counting the remaining intact nuclei. Survival is reported relative to untreated controls and is the mean \pm SEM $(n=3)$. Addition of 3-MA (added $8 \mathrm{hr}$ after treatment with camptothecin and BAF) or bafilomycin had no effect on caspase-independent death. This is representative of three independent experiments.

Reactive oxygen species are generated in caspase-independent neuronal death, and the antioxidant Tempol offers protection against neuronal death

Mitochondrial dysfunction has been described to lead to the generation of ROS, which could be a contributing factor to cell death (Zamzami et al., 1995; Green and Reed, 1998). Therefore, we first investigated whether ROS are generated in our model of caspaseindependent cell death. To assess ROS generation, we used the fluorescent dyes DHE and DCF. In the case of DHE labeling, we concurrently labeled the cultures with the viability dye calcein AM to ensure that the neurons counted as showing ROS generation were still viable. We found that a significant percentage of neurons treated with the combination of camptothecin and BAF accumulated ROS over time (Fig. $3 A, C$ ). The time course of ROS accumulation as shown in Figure $3 C$ in parallel with the time course of neuronal death showed that this was a relatively late event and correlated closely with the onset of death in the cultures. Similar results were obtained qualitatively with DCF labeling (Fig. 3B). The neurons labeled with DCF still have an intact outer membrane as shown by exclusion of ethidium homodimer but, on phase, show signs of advanced degeneration.

We then investigated whether antioxidant pharmacological agents would have a protective effect in this caspase-independent death. A range of common antioxidants, including vitamin E, Trolox, NAC, GSH-MEE, Tiron, MnTBAP, or MnTMPyP, used at standard concentrations (see Materials and Methods) had no effect on survival. Representative data from an experiment with vitamin $\mathrm{E}$ are shown in Figure $4 A$. In parallel experiments, we examined whether such antioxidants prevented the generation of ROS. Surprisingly, we found that they did not, even when high concentrations had been used (see range of concentrations used in Materials and Methods) or when they had been re-added after $10 \mathrm{hr}$ of treatment (see Fig. $4 A$ for representative experiment with vitamin $\mathrm{E}$ ). These data raised the possibility that the antioxidant 

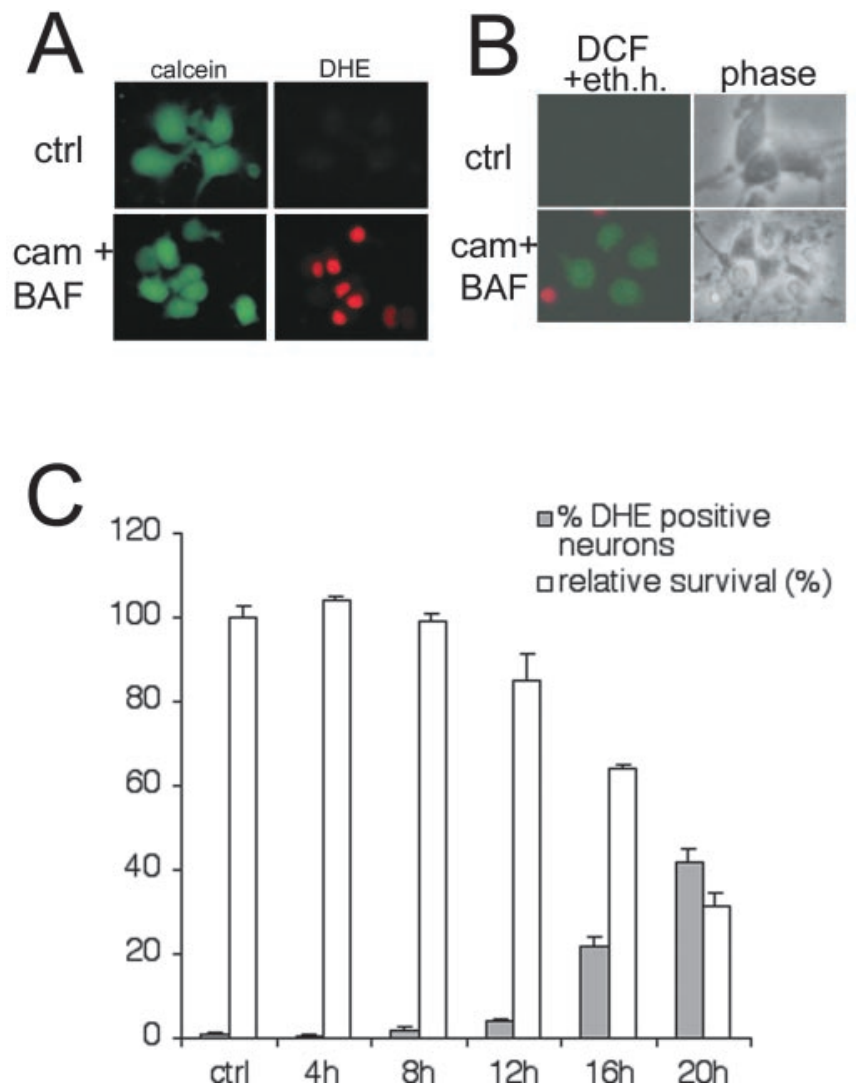

Figure 3. ROS are generated during caspase-independent death. $A, B$, E18 cortical neuron cultures were treated with no additives (ctrl) or with the combination of camptothecin and BAF (cam + BAF) for $20 \mathrm{hr}$. Generation of ROS was assessed by incubation with the fluorescent dye DHE (red). Neurons were counterstained with the green fluorescent viability indicator calcein $A M(A)$. Alternatively, the green fluorescing probe DCF in combination with the death indicator ethidium homodimer (eth. h., red) was used (B). The percentage of DHE-positive neurons relative to live (calcein AM-positive) neurons was counted over time. In parallel, neuronal survival was assessed by nuclear counts. Numbers are expressed as mean $\pm \operatorname{SEM}(n=3)$.
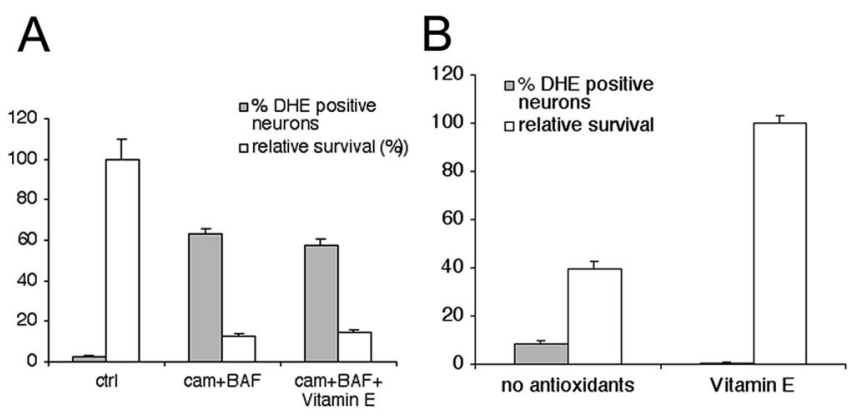

Figure 4. Common antioxidants represented here by vitamin $E$ do not offer any protection. $A$, Cortical neurons were treated with camptothecin and BAF (cam + BAF) for $20 \mathrm{hr}$. Concomitant treatment with the antioxidant vitamin $\mathrm{E}(100 \mu \mathrm{m})$, which was re-added after $12 \mathrm{hr}$, did not have an effect on survival, as assessed by nuclear counts, and also failed to protect against ROS generation (percentage of DHE-positive neurons relative to live neurons). In contrast, vitamin E was effective in preventing the ROS generation and death in neurons plated and cultured for $48 \mathrm{hr}$ in antioxidant-free medium $(B)$.

treatments used may not be effective as antioxidants in our system. We thus tested these substances in another model of oxidative stress, in which neuronal death was induced by culturing the neurons for $48 \mathrm{hr}$ in antioxidant-free medium (neurobasal medium plus antioxidant-free B-27 supplement). All of these agents were effective in preventing the generation of ROS and neuronal
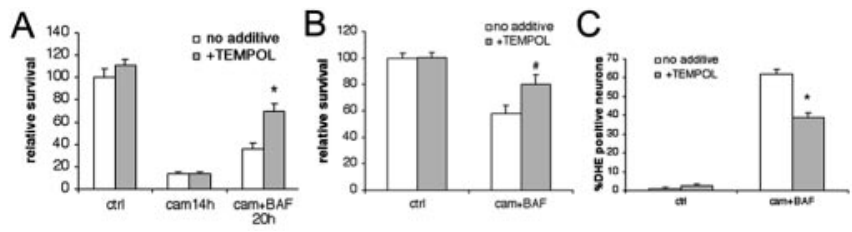

Figure 5. The free-radical scavenger Tempol is effective in preventing ROS generation and neuronal death. Cortical neurons were treated with cam alone or camptothecin plus BAF. $A$, The addition of Tempol $(5 \mathrm{~mm}) 10 \mathrm{hr}$ after the onset of treatment had no effect on survival in neurons treated with camptothecin alone (nuclear counts after $14 \mathrm{hr}$ ) but significantly improved survival in neurons treated with camptothecin and BAF as assessed by nuclear counts after $20 \mathrm{hr}$. $B$, Counts of live (calcein AM-positive) neurons in a defined number of fields in each well confirmed the protective effect of Tempol. $C$, In parallel, ROS generation reported as the percentage of DHE-positive neurons relative to live neurons was assessed. All counts were conducted in a blinded manner. Numbers are reported as the mean $\pm \operatorname{SEM}\left(A, B, n=4 ; C, n=3 ;{ }^{*} p<0.01\right.$; $\# p<0.05$; Student's $t$ test).

death in this model (see Fig. $4 B$ for representative experiment with vitamin $\mathrm{E}$ ).

As an alternative approach to a pharmacological reduction of oxidative stress, we overexpressed the antioxidant protein human SOD1 in the cultures and again obtained no effect on either ROS generation or neuronal death (data not shown). Because the B-27 supplement routinely used in the cultures already contains antioxidants, we hypothesized that a reason for the lack of effect of antioxidants on caspase-independent ROS generation may be that there is already a saturating concentration of antioxidants in the medium. We therefore performed the same experiments in neurobasal medium plus antioxidant-free B-27 supplement with $2.5 \mu \mathrm{g} / \mathrm{ml}$ of vitamin $\mathrm{E}$, which was the minimum concentration that maintained viability of control cultures for several days. Even in these conditions, the addition of vitamin $\mathrm{E}(100 \mu \mathrm{g} / \mathrm{ml})$ or MnTBAP had no effect on ROS generation or survival of neurons exposed to camptothecin and BAF (data not shown).

We next cotreated our cultures with the substance Tempol, a cell permeable nitroxide that acts as a free radical scavenger and spin trap agent (Cuzzocrea et al., 2000; Thiemermann et al., 2001). Because Tempol was effective in preventing caspasedependent cell death, it was added to the cultures after $10 \mathrm{hr}$ of treatment with camptothecin and BAF. At this time point, the majority of the neurons treated with camptothecin alone had undergone cell death, and no additional protection could be achieved by the addition of Tempol (Fig. 5A). However, caspaseindependent cell death was significantly abrogated by Tempol, even when added in a delayed manner (Fig. 5A,B). This result was obtained by counting both the intact nuclei of lysed neurons (Fig. 5A) and calcein AM-positive neurons in the culture dishes (Fig. $5 B$ ). The discrepancy in the absolute number of surviving neurons between these two methods is likely attributable to the fact that nuclear alterations occur before the disruption of the cellular membrane, and thus neurons with a damaged nucleus would still be counted as calcein AM positive. We next assessed the oxidative activity in neurons and found that Tempol reduced the generation of ROS, as indicated by DHE labeling (Fig. 5C).

\section{AIF and the mitochondrial permeability transition pore are} not involved in caspase-independent neuronal death

AIF is a mitochondrial death effector that is released from the intermembrane space after certain death stimuli. It then translocates to the nucleus, where it induces large-scale DNA fragmentation and chromatin condensation (Susin et al., 1999; Daugas et al., 2000). Although some studies report that AIF is released after 

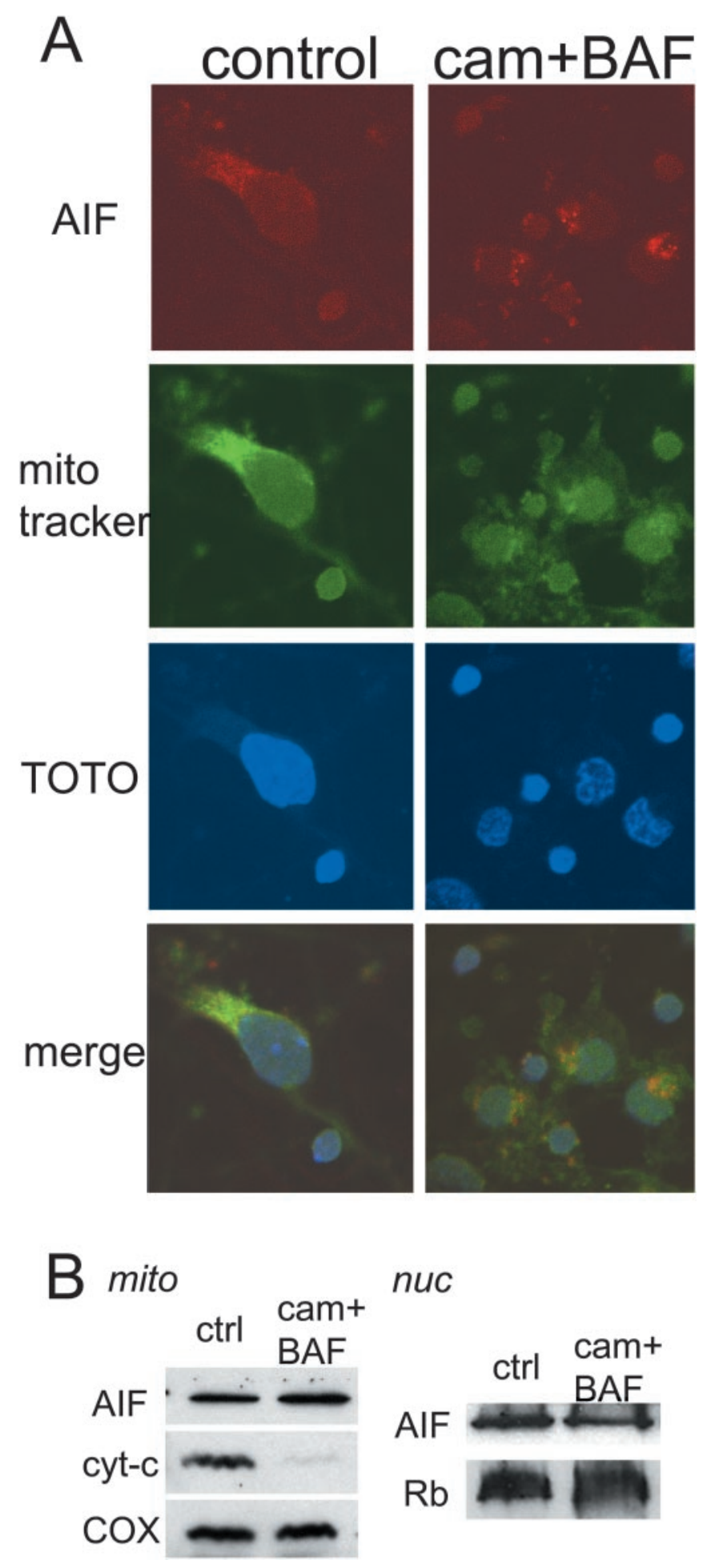

nuc

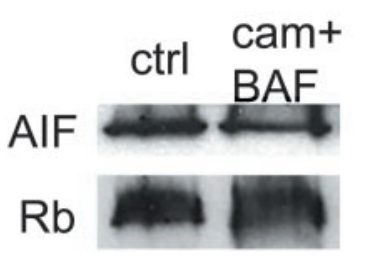

Figure 6. There is no obvious translocation of AlF from the mitochondria to the nucleus in caspase-independent cell death. $A$, After treatment with no additives (control) or with camptothecin and BAF (cam + BAF) for $20 \mathrm{hr}$, the neurons were stained with Mitotracker Green to label mitochondria. After fixation, neurons were immunostained with an anti-AlF monoclonal antibody and counterstained with the nuclear dye TOTO. Confocal imaging shows colocalization of AIF with Mitotracker Green in controls as well as treated cells. There was no nuclear AIF translocation observed, even in frankly degenerating cells. The reduction of Mitotracker Green in treated cells is likely attributable to some loss of mitochondria (Fig. 2A). B, Cultured E18 neurons were treated with camptothecin and BAF (cam +BAF) for $20 \mathrm{hr}$, and subcellular fractions were obtained by differential centrifugation. Proteins from the enriched mitochondrial fraction (mito; $40 \mu \mathrm{g}$ ) and the enriched nuclear fraction (nuc; $20 \mu \mathrm{l}$ ) were then separated by SDS-PAGE, blotted to nitrocellulose membranes, and probed with the indicated antibodies.
A
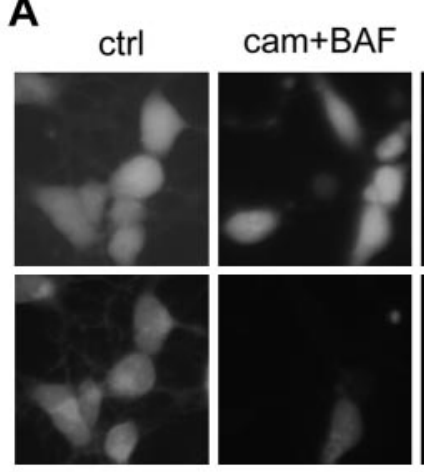

cam+BAF
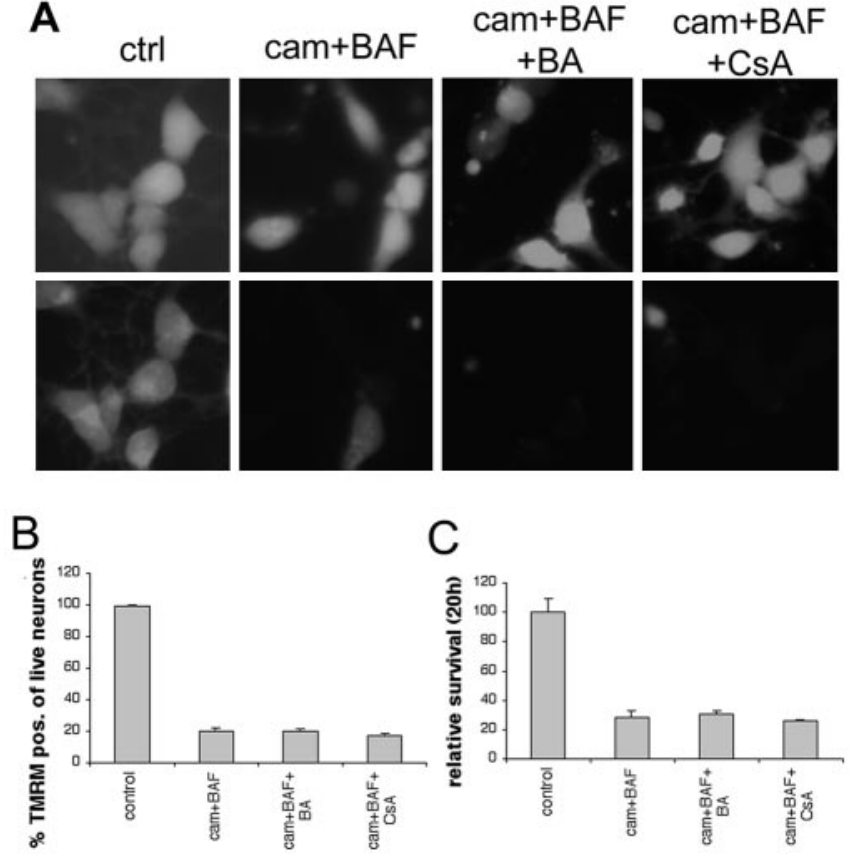

Figure 7. Inhibitors of the mitochondrial PTP do not protect against loss of mitochondrial transmembrane potential or neuronal death. E18 cortical neurons were treated with no additives (control), camptothecin and BAF (cam + BAF) alone, or in combination with the mitochondrial PTP inhibitors bonkgrekic acid $(B A ; 5 \mu \mathrm{M})$ or cyclosporin $A(C s A ; 1 \mu \mathrm{M})$. Mitochondrial transmembrane potential was assessed after $20 \mathrm{hr}$ by TMRM labeling $(A$, bottom row). Neurons were counterstained with the viability indicator calcein $A M(A$, top row). $B$, The percentage of live (calcein AM-positive) neurons that maintained TMRM labeling in each condition was assessed under a fluorescence microscope. C, Survival was assessed in parallel by nuclear counts. In both cases, numbers are reported as mean $\pm \operatorname{SEM}(n=3)$. This is representative of three independent experiments.

cytochrome $c$ in a caspase-dependent manner (Arnoult et al., 2002, 2003; Wang et al., 2002), it has also been shown to translocate independently of caspase activation (Susin et al., 1999; Daugas et al., 2000; Cregan et al., 2002). It thus seemed a possible candidate as a death effector in our caspase-independent cell death model. We first used immunostaining with an AIF antibody, concurrently with labeling with the mitochondrial marker Mitotracker Green and a nuclear dye. We found no evidence of translocation of AIF to the nucleus $20 \mathrm{hr}$ after treatment with camptothecin and BAF (Fig. 6A). This result was confirmed through the use of another AIF antibody, a rabbit polyclonal antiserum generously provided by Drs. Kroemer and Susin (data not shown). In addition, Western immunoblotting of nuclear and mitochondrial fractions showed that, although cytochrome $c$ release occurred from the mitochondria in caspase-independent death, there was no loss of AIF from the mitochondria, and there was no accumulation of AIF in the nuclear fraction (Fig. $6 B$ ).

Opening of the mitochondrial permeability transition pore (PTP) was recently proposed as a mechanism accounting for caspase-independent death of sympathetic neurons (Chang and Johnson, 2002). We therefore investigated whether inhibitors of the PTP would affect loss of MTP or survival in caspase-

\section{$\leftarrow$}

There is clear loss of cytochrome c but not of AIF in the mitochondrial fraction. There is no accumulation of AIF in the nuclear fraction during the time tested. PRb and COX are used as markers of the nuclear and mitochondrial fractions, respectively, and as controls for protein loading. 

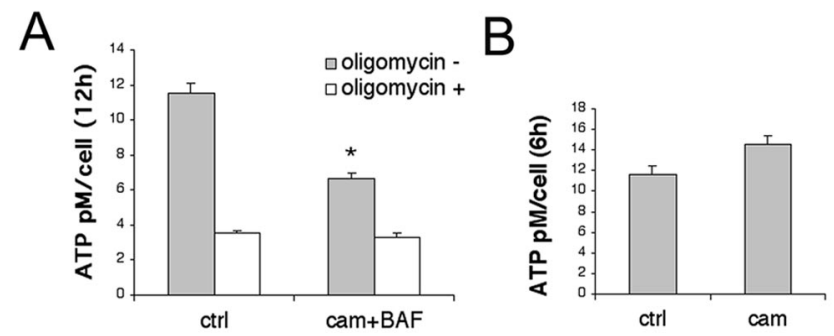

Figure 8. A specific decrease of mitochondria-derived ATP occurs in caspase-independent death. $A$, E18 cortical neurons were treated with no additives (ctrl) or with camptothecin and BAF (cam + BAF) for $12 \mathrm{hr}$. Cells were then harvested for ATP measurements and parallel assessment of survival by nuclear counts. ATP levels were measured with a luminometric assay kit (Roche Products), normalized to cell numbers, and represented as the mean \pm SEM $(n=3)$. To assess ATP that was not derived from the mitochondria, neurons were treated with oligomycin $(2 \mu \mathrm{g} / \mathrm{ml})$ for $15 \mathrm{~min}$ before ATP measurements. Although the nonmitochondrial ATP (white bars) did not change, there was a significant decrease of total ATP content in cultures treated with cam +BAF compared with controls (gray bars; ${ }^{* *} p<0.001$; Student's $t$ test). $B$, E18 cortical neurons were treated for $6 \mathrm{hr}$ with no additives (ctrl) or with $10 \mu \mathrm{m}$ cam, and total ATP content was assessed as above. There was no difference in ATP content between the two conditions. These are representative of three independent experiments, all performed in a blinded manner.

independent death. We found no effect of two different PTP inhibitors, cyclosporin A or bongkrekic acid, on either parameter (Fig. 7A-C).

\section{Mitochondrial ATP levels are diminished specifically in caspase-independent neuronal death}

One consequence of the loss of cytochrome $c$ can be the disruption of the mitochondrial respiratory chain, in particular at the level of complexes III and IV and, consequently, of the energy metabolism of the cell. Although a drop in ATP production has been observed in apoptotic death, it seems to occur fairly late and is not generally considered a mechanism for induction of apoptosis (Bossy-Wetzel et al., 1998; Green and Reed, 1998). Presumably, caspase activation and execution of apoptotic cell death occur very shortly after cytochrome $c$ release, before a drop in energy levels leads to cellular dysfunction and death (Green and Kroemer, 1998). However, in a setting where caspases are inhibited, cells may remain alive with dysfunctional mitochondria for more prolonged periods of time. As we have shown previously, $>60 \%$ of neurons treated with camptothecin and BAF show mitochondrial cytochrome $c$ release, and $>30 \%$ lose mitochondrial transmembrane potential after only $10 \mathrm{hr}$ of treatment. At this time point, little if any cell death has occurred (Stefanis et al., 1999).

We therefore analyzed the cellular energy levels at a similar time point in neurons treated with camptothecin and BAF. ATP levels were measured by a luminometric assay and were normalized to cell number. After $12 \mathrm{hr}$ of treatment, cellular ATP levels dropped by $>40 \%$ compared with controls (Fig. $8 \mathrm{~A}$ ). Almost $70 \%$ of cellular ATP levels in control cultures was inhibited by oligomycin, and was therefore of mitochondrial origin (Fig. 8A). Nonmitochondrial ATP levels were similar in controls and in neurons treated with camptothecin and BAF, indicating that the ATP reduction in the treated neurons occurs in the mitochondria-generated ATP (Fig. 8A). To ensure that energy depletion occurs specifically in caspase-independent death, we also measured ATP levels in neurons treated with camptothecin alone. After $6 \mathrm{hr}$ of treatment, at a time point when caspases are activated and survival levels are similar to those observed after 12

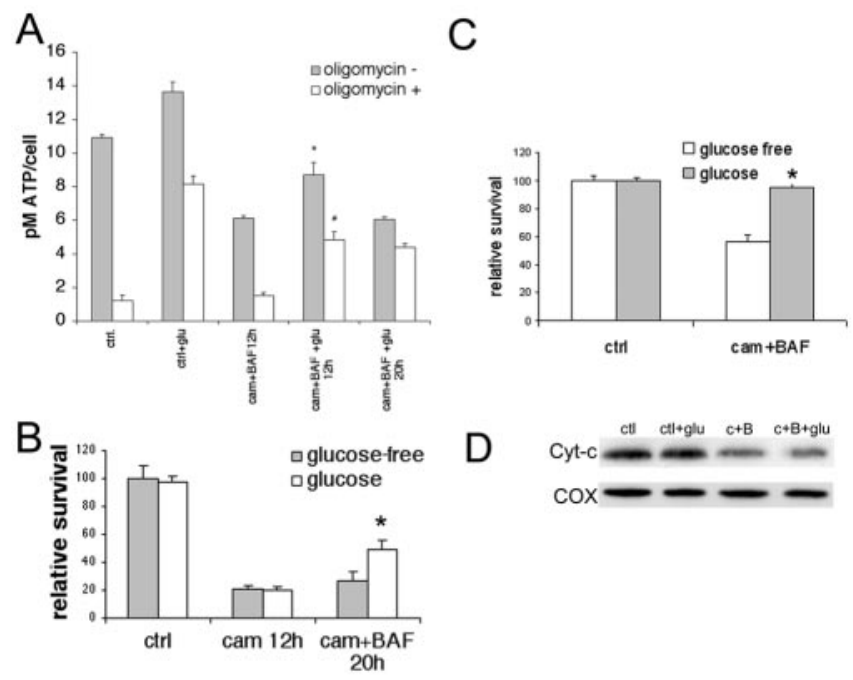

Figure 9. Modulation of nonmitochondrial source of ATP leads to a specific increase of survival in caspase-independent death. $A$, E18 cortical neurons were plated and cultured in glucose-free RPMI medium containing $5 \mathrm{~mm}$ pyruvate and the supplement B-27. The cells were exposed to no additives (ctrl), $4 \mathrm{mg} / \mathrm{ml} \mathrm{D}$-glucose (ctrl + glu), or $10 \mu \mathrm{m}$ camptothecin and 100 $\mu \mathrm{M} B A F(\mathrm{cam}+\mathrm{BAF})$ or glucose, camptothecin, and BAF (cam $+B A F+$ glu). Measurements of ATP levels were obtained with (white bars) or without (gray bars) a 15 min pretreatment with oligomycin. As in Figure $8 A$, there was a decrease of total ATP content with the cam and BAF treatment in the glucose-free medium after $12 \mathrm{hr}$ of treatment. The addition of glucose significantly augmented total ATP content in cultures treated with cam and BAF $\left({ }^{*} p<0.05\right)$ by increasing the nonmitochondrial ATP (\#p $<0.01$ ). However, the increase in total ATP by the addition of glucose was not sustained over time, as shown by the measurements at $20 \mathrm{hr}$. B, E18 cortical neurons were plated and cultured in glucose-free medium. They were then treated with no additives (ctrl), camptothecin for $12 \mathrm{hr}$ (cam $12 \mathrm{hr}$ ), or cam and BAF for $20 \mathrm{hr}$ in the presence or absence of $4 \mathrm{mg} / \mathrm{ml} \mathrm{D}$-glucose. The presence of glucose (white bars) offered no survival advantage to control cells or to those exposed to camptothecin but significantly attenuated caspase-independent cell death $\left({ }^{*} p<0.05\right)$. C, Counts of live (calcein AM-positive) neurons in a defined number of fields in each well confirmed the protective effect of glucose in caspaseindependent cell death $\left({ }^{*} p<0.01\right)$. D, Neurons were cultured in glucose-free medium as above and were then treated for $16 \mathrm{hr}$ with no additives $(\mathrm{ct})$ ), glucose $(\mathrm{ctl}+\mathrm{glu})$, camptothecin and $B A F(C+B)$, or camptothecin and BAF plus glucose $(C+B+g l u)$. Proteins from enriched mitochondrial fractions were then obtained by differential centrifugation of the cell lysates and analyzed by immunoblotting with indicated antibodies. The addition of glucose had no effect on mitochondrial cytochrome c content in either control conditions or neurons treated with camptothecin and BAF. COX is used as a marker for protein loading. This is representative of two independent experiments and shows similar results as two additional experiments in which lysates were collected after $12 \mathrm{hr}$ of treatment.

hr of combined camptothecin and BAF treatment, ATP levels were not diminished compared with controls (Fig. 8B).

\section{Manipulation of cellular ATP levels alters caspase-independent death}

Having shown that there was a relative depletion of cellular ATP levels in caspase-independent death, we investigated whether providing neurons with an alternative source of ATP, namely glycolysis, would have an affect on the cellular energy state and cell death. Because the neurobasal medium that we used in previous experiments contains high amounts of glucose, we performed experiments to address this issue in a glucose-free medium supplemented with pyruvate. Neurons survive in this medium for several days. The reduction of mitochondrial ATP production observed in caspase-independent death was reproduced under these glucose-free culture conditions. The addition of glucose to the medium simultaneously with camptothecin and BAF led to a significant increase of cellular ATP levels $12 \mathrm{hr}$ later. As shown by inhibition of mitochondrial ATP production by 
oligomycin, this increase in ATP levels was attributable to nonmitochondrial sources (Fig. 9A). However, this increase in ATP levels was not sustained over time: $20 \mathrm{hr}$ after treatment, ATP content dropped and reached the levels observed in neurons not supplemented with glucose after $12 \mathrm{hr}$ of treatment with camptothecin and BAF. This drop in ATP production was primarily attributable to an additional loss of mitochondrial ATP.

We then evaluated the effects of this increase of cellular ATP levels on caspase-independent death. After $20 \mathrm{hr}$ of treatment, we found an $\sim 80 \%$ increase in survival of neurons supplemented with glucose: $27 \pm 6.4 \%$ of neurons survived in the glucose-free condition, whereas $49 \pm 7 \%$ survived in the glucose-containing medium (Fig. 9B). This survival effect was specific because apoptotic cell death induced by camptothecin alone was not affected (Fig. 9B). The beneficial effect of glucose on survival in caspaseindependent death was confirmed by counts of calcein AMpositive neurons in the culture dishes (Fig. 9C). Again, as in the case of antioxidant treatment (Fig. $5 B$ ), the absolute numbers of surviving neurons were found to be higher with this method.

Because it has been reported that high concentrations of glucose might lead to alterations in the levels of bcl-2 protein family members and thus could prevent cytochrome $c$ release (Schaffer et al., 2000; Pampfer et al., 2001; Sakuma et al., 2002), we investigated whether the addition of glucose would affect loss of mitochondrial cytochrome $c$ in our model. Immunoblots of cytochrome $c$ in crude mitochondrial fractions of cell lysates were performed at two different time points after treatment (two Western blots at 12 or $16 \mathrm{hr}$ each) and showed no effect on mitochondrial cytochrome $c$ release with glucose supplementation (Fig. 9D).

\section{Increasing intracellular ATP and reducing oxidative stress leads to an additive increase in neuronal survival}

Because both increasing intracellular ATP by supplementation with glucose and reducing free-radical generation by the addition of the antioxidant Tempol had shown beneficial effects on neuronal survival, we investigated whether combining the strategies could lead to an additive effect. In two different experiments, neurons were treated for $20 \mathrm{hr}$ as above with and without glucose and with and without the addition of Tempol. Although supplementation of glucose alone increased survival by $\sim 90 \%$ and the addition of Tempol alone increased survival by $\sim 70 \%$, the combination of both treatments led to a 2.6-fold increase in survival. However, many neurons still died in these experiments, even with the combined treatment of Tempol and glucose (data not shown).

\section{Discussion}

Caspase-independent death has recently been recognized as a potential limiting factor in the usefulness of caspase-directed therapies for neurological diseases (for review, see Rideout and Stefanis, 2001). We (Stefanis et al., 1999; Keramaris et al., 2000) and others (Xue et al., 1999; Chang and Johnson, 2002; Cregan et al., 2002) have been studying the mechanisms through which such neuronal cell death occurs, and have suggested that alterations at the level of the mitochondria, apart from their better established pro-apoptotic effects, may also lead to caspaseindependent death. Identification of such mechanisms may lead to combined treatment approaches that are more effective than caspase inhibition alone and may offer insights into the way neurons degenerate in the absence of apparent caspase activation in a number of neurodegenerative conditions (Stefanis et al., 1999; Rideout and Stefanis, 2001).

\section{Mitochondrial energy depletion is a mechanism of caspase- independent neuronal death}

A major finding of the current work is that a specific decrease of mitochondrially generated ATP occurs in cultured rat cortical neurons treated with the combination of camptothecin and a general caspase inhibitor. More importantly, manipulation of cellular ATP levels leads to enhanced survival in this model of caspase-independent neuronal death. Thus, caspaseindependent neuronal death is, at least in part, attributable to energy depletion.

Such mitochondrial energy depletion and subsequent death may occur secondary to caspase-independent cytochrome $c$ release (Stefanis et al., 1999) and the ensuing loss of function of complexes III and IV of the respiratory chain. Consistent with this, caspase-independent redistribution of cytochrome $c$ leads to mitochondrial respiratory dysfunction in Fas-stimulated hepatocytes (Mootha et al., 2001), and treatment of cortical neurons with the complex III inhibitor antimycin A leads to caspaseindependent death (Stefanis et al., 1999).

Another event that occurs in caspase-independent neuronal death is the loss of MTP. This, unlike cytochrome $c$ translocation, occurs in a slightly delayed manner in the presence of caspase inhibitors, implying that caspases may be partially involved in MTP loss, perhaps in a positive feedback loop (Stefanis et al., 1999). A caveat is that the cationic dyes that we used may also be sensitive to changes in plasma membrane potential, the loss of which was recently demonstrated to be caspase-dependent (Dussmann et al., 2003). Consistent with the idea that caspases partially affect MTP, caspase- 3 inhibits the respiratory chain and leads to loss of MTP (Ricci et al., 2003). MTP loss does eventually occur in our model and is nearly complete by $20 \mathrm{hr}$ (Stefanis et al., 1999) (Fig. $7 A, B$ ). The inability to maintain a proton gradient across the mitochondrial membrane may be the result of impaired electron transport through the dysfunctional respiratory chain and may contribute to mitochondrial energy failure.

To counteract the decline in cellular ATP content, we used glucose, which would be expected to lead to an increase of ATP production through glycolysis and not through the malfunctioning mitochondria. Such an increase in nonmitochondrial ATP was indeed observed and led to enhanced survival specifically in caspase-independent death. It should be noted however that this strategy was only partially and transiently effective in restoring cellular ATP levels. It remains to be seen whether other strategies aimed at restoring the energy state of the neurons may be more effective in preventing neuronal death.

\section{A free-radical scavenger protects against caspase-independent death}

ROS may kill in a caspase-independent manner and have been suggested as ancestral mediators of programmed cell death in organisms that lack caspases (for review, see Fleury et al., 2002). Because mitochondrial alterations and, in particular, loss of cytochrome $c$ have been shown to induce ROS generation (Cai and Jones, 1998), we investigated the role of ROS in our model. We found increased generation of ROS in caspase-independent death; however, this was a relatively late event, occurring close to the time of cell death. The substance Tempol, a nitroxide acting as free-radical scavenger and spin trap agent (Cuzzocrea et al., 2000; Thiemermann et al., 2001), diminished oxidative stress and improved survival. However, many common antioxidants, as well as the overexpression of SOD, had no effect on neuronal death or ROS generation, although they were effective in another model of oxidative stress. Why these antioxidant treatments were not ef- 
fective is yet unclear. It could be that they did not penetrate the cell well or that they were not able to withstand the high levels of oxidative stress present within the cell, whereas Tempol as a free radical scavenger had a better effect. Alternatively, superoxide may react with $\mathrm{NO}$ to form peroxynitrite at a faster rate than it is processed to $\mathrm{H}_{2} \mathrm{O}_{2}$ by SOD, especially under conditions of enhanced NO formation (Dyckens 1997; Radi et al., 2002). In addition, peroxynitrite reacts with the Mn center of SOD, leading to its inactivation and a subsequent decrease in $\mathrm{O}_{2}$ dismutation, thus initiating a positive feedback cycle (Radi et al., 2002). Potentially, the more "classic" antioxidants and SOD treatment are not efficient enough to disrupt this reaction. Tempol can efficiently scavenge peroxynitrite and other nitrogen-derived free radicals (Thiemermann et al., 2001; Bonini et al., 2002) and thus may lead to interruption of this feedback loop. Alternatively, NO produced from the neurons or NO products like nitrites contained in the cell culture medium could react with $\mathrm{O}_{2}$ and form radicals that become fatal in neurons already compromised in their antioxidant defenses.

\section{Autophagy and lysosomal activity are increased but are not instrumental in caspase-independent neuronal cell death} Autophagy is not only a normal response of hepatocytes to nutrient deprivation but also a specific type of developmental neuronal cell death (Mortimore and Poso, 1986; Clarke, 1990). Autophagy may thus represent an alternative form of programmed cell death (Clarke, 1990; Xue et al., 1999; Saeki et al., 2000). We show here that autophagy is activated in caspase-independent death of cultured E18 cortical neurons and that it leads to a general activation of the lysosomal pathway. However, autophagy is not instrumental to caspase-independent neuronal death in our model, because inhibitors of autophagy or of the vacuolar ATPase were not protective. This is in contrast to the findings of Xue et al. (1999), who found that 3-MA protected against rat sympathetic neuron caspase-independent death. In the same model, mitochondria were selectively eliminated by an autophagic process (Xue et al., 2001). Although some mitochondria were sequestered in autophagic vacuoles and numbers of mitochondria were found to be diminished (Fig. $2 A$ ), complete elimination of mitochondria does not occur in our model. After $20 \mathrm{hr}$ of treatment with camptothecin and BAF, intact mitochondrial profiles were still found (Fig. 1C), and COX IV protein levels were preserved (Fig. $6 B$ ). The difference in the fate of mitochondria between these two models might account for, in part, the importance of autophagy in eliciting neuronal death. It is possible that in our model, the temporal relationship between loss of mitochondrial function and autophagic sequestration is different from the model of Xue et al. $(1999,2001)$ and, thus, neuronal death might occur through energy depletion, free radical generation, and potentially other mechanisms before cell death resulting from autophagy occurs.

\section{AIF translocation and PTP opening are not involved in caspase-independent neuronal death}

In contrast to a recent study using a very similar model of caspase-independent neuronal death in which camptothecin was administered to mouse cortical neurons lacking apoptotic protease activating factor-1 (Apaf-1) (Cregan et al., 2002), we found no evidence for translocation of the mitochondrial death mediator AIF from the mitochondria to the nucleus. There are several differences between our study and that of Cregan et al. (2002) that could account for this discrepancy: species differences, different dissection and culture protocols, and a different temporal evolution of caspase-independent death. Alternatively, compensatory pathways, such as upregulation of an AIF death pathway, may be activated in Apaf-1 knock-out mice. Consistent with this idea, the caspase $9 / 3$ pathway shows compensatory activation in caspase 2 knock-out mice (Troy et al., 2001).

Our findings of the lack of PTP involvement in our model contrast with another recent study in which administration of 10 $\mu \mathrm{M}$ of the PTP inhibitor cyclosporin A had selective survival effects on caspase-independent but not caspase-dependent death of NGF-deprived rat sympathetic neurons (Chang and Johnson, 2002). The dose of cyclosporin A used was 10-fold higher than in our study. We were unable to use this agent at such high concentrations because of its toxicity (data not shown); however, in a previous study, cyclosporin A exerted effects on mitochondrial alterations and neuronal death at concentrations as low as $200 \mathrm{~nm}$ (Khaspekov et al., 1999). Furthermore, another PTP inhibitor, bongkrekic acid, used at concentrations as high as $50 \mu \mathrm{M}$ also failed to prevent loss of transmembrane potential and neuronal death. We conclude that the loss of MTP occurs through another mechanism and that opening of the PTP is not required for caspase-independent death in our model.

In conclusion, our present study provides evidence that autophagy is the morphological form of caspase-independent neuronal death. This death occurs, at least in part, because of energy depletion and generation of free radicals. The survivalpromoting effect of increasing cellular ATP levels and reducing free radicals is additive, suggesting different mechanisms of action. So far, in caspase-independent neuronal death, more attention has been paid to specific molecules that may be released by the mitochondria in a caspase-independent manner (Chang and Johnson, 2002; Cregan et al., 2002). We cannot exclude that other potential mediators might also be a factor in our model of caspase-independent death, especially because combined treatment targeted against both energy depletion and free radical generation did not lead to complete survival. However, our findings argue that the general energy and redox state of the neurons play a critical role. Antioxidant treatment in combination with an optimized delivery of alternative sources of energy to neurons that are temporarily saved by caspase inhibition would appear to be a valuable therapeutic strategy, especially in situations in which the death stimulus continues to be present.

\section{References}

Arnoult D, Parone P, Martinou JC, Antonsson B, Estaquier J, Ameisen JC (2002) Mitochondrial release of apoptosis-inducing factor occurs downstream of cytochrome c release in response to several proapoptotic stimuli. J Cell Biol 159:923-929.

Arnoult D, Gaume B, Karbowski M, Sharpe JC, Cecconi F, Youle RJ (2003) Mitochondrial release of AIF and EndoG requires caspase activation downstream of Bax/Bak-mediated permeabilization. EMBO J 22:4385-4399.

Bonini MG, Mason RP, Augusto O (2002) The mechanism by which 4-hydroxy-2, 2, 6, 6-tetramethylpiperidene-1-oxyl (tempol) diverts peroxynitrite decomposition from nitrating to nitrosating species. Chem Res Toxicol 15:506-511.

Bossy-Wetzel E, Newmeyer DD, Green DR (1998) Mitochondrial cytochrome $\mathrm{c}$ release in apoptosis occurs upstream of DEVD-specific caspase activation and independently of mitochondrial transmembrane depolarization. EMBO J 17:37-49.

Bowman EJ, Siebers A, Altendorf K (1988) Bafilomycins: a class of inhibitors of membrane ATPases from microorganisms, animal cells, and plant cells. Proc Natl Acad Sci USA 85:7972-7976.

Cai J, Jones DP (1998) Superoxide in apoptosis. Mitochondrial generation triggered by cytochrome c loss. J Biol Chem 273:11401-11404.

Cao G, Luo Y, Nagayama T, Pei W, Stetler RA, Graham SH, Chen J (2002) Cloning and characterization of rat caspase-9: implications for a role in 
mediating caspase- 3 activation and hippocampal cell death after transient cerebral ischemia. J Cereb Blood Flow Metab 22:534-546.

Chang LK, Johnson Jr EM (2002) Cyclosporin A inhibits caspaseindependent death of NGF-deprived sympathetic neurons: a potential role for mitochondrial permeability transition. J Cell Biol 157:771-778.

Clarke PG (1990) Developmental cell death: morphological diversity and multiple mechanisms. Anat Embryol (Berl) 181:195-213.

Cregan SP, Fortin A, MacLaurin JG, Callaghan SM, Cecconi F, Yu SW, Dawson TM, Dawson VL, Park DS, Kroemer G, Slack RS (2002) Apoptosis-inducing factor is involved in the regulation of caspaseindependent neuronal cell death. J Cell Biol 158:507-517.

Cutillas B, Espejo M, Gil J, Ferrer I, Ambrosio S (1999) Caspase inhibition protects nigral neurons against 6-OHDA-induced retrograde degeneration. NeuroReport 10:2605-2608.

Cuzzocrea S, McDonald MC, Mazzon E, Siriwardena D, Costantino G, Fulia F, Cucinotta G, Gitto E, Cordaro S, Barberi I, De Sarro A, Caputi AP, Thiemermann C (2000) Effects of tempol, a membrane-permeable radical scavenger in a gerbil model of brain injury. Brain Res 875:96-106.

Daugas E, Susin SA, Zamzami N, Ferri KF, Irinopoulou T, Larochette N, Prevost MC, Leber B, Andrews D, Penninger J, Kroemer G (2000) Mitochondrio-nuclear translocation of AIF in apoptosis and necrosis. FASEB J 14:729-739.

Dussmann H, Rehm M, Kogel D, Prehn JH (2003) Outer mitochondrial membrane permeabilization during apoptosis triggers caspaseindependent mitochondrial and caspase-dependent plasma membrane potential depolarization: a single-cell analysis. J Cell Sci 116:525-536.

Dyckens J (1997) Mitochondrial free radical production and oxidative pathophysiology: implication for neurodegenerative disease. In: Mitochondria and free radicals in neurodegenerative diseases (Beal MF, Howell N, Bodis-Wollner I, eds), pp 29-55. New York: Wiley-Liss.

Elmore SP, Qian T, Grissom SF, Lemasters JJ (2001) The mitochondrial permeability transition initiates autophagy in rat hepatocytes. FASEB J 15:2286-2287.

Fleury C, Mignotte B, Vayssiere JL (2002) Mitochondrial reactive oxygen species in cell death signaling. Biochimie 84:131-141.

Friedman WJ, Ibanez CF, Hallbook F, Persson H, Cain LD, Dreyfus CF, Black IB (1993) Differential actions of neurotrophins in the locus coeruleus and basal forebrain. Exp Neurol 119:72-78.

Green D, Kroemer G (1998) The central executioners of apoptosis: caspases or mitochondria? Trends Cell Biol 8:267-271.

Green DR, Reed JC (1998) Mitochondria and apoptosis. Science 281:1309-1312.

Hsu LJ, Sagara Y, Arroyo A, Rockenstein E, Sisk A, Mallory M, Wong J, Takenouchi T, Hashimoto M, Masliah E (2000) alpha-synuclein promotes mitochondrial deficit and oxidative stress. Am J Pathol 157:401-410.

Keramaris E, Stefanis L, MacLaurin J, Harada N, Takaku K, Ishikawa T, Taketo MM, Robertson GS, Nicholson DW, Slack RS, Park DS (2000) Involvement of caspase 3 in apoptotic death of cortical neurons evoked by DNA damage. Mol Cell Neurosci 15:368-379.

Khaspekov L, Friberg H, Halestrap A, Viktorov I, Wieloch T (1999) Cyclosporin $\mathrm{A}$ and its nonimmunosuppressive analogue N-Me-Val-4cyclosporin A mitigate glucose/oxygen deprivation-induced damage to rat cultured hippocampal neurons. Eur J Neurosci 11:3194-3198.

Knoblach SM, Nikolaeva M, Huang X, Fan L, Krajewski S, Reed JC, Faden AI (2002) Multiple caspases are activated after traumatic brain injury: evidence for involvement in functional outcome. J Neurotrauma 19:1155-1170.

Krohn AJ, Preis E, Prehn JH (1998) Staurosporine-induced apoptosis of cultured rat hippocampal neurons involves caspase-1-like proteases as upstream initiators and increased production of superoxide as a main downstream effector. J Neurosci 18:8186-8197.

Larsen KE, Sulzer D (2002) Autophagy in neurons: a review. Histol Histopathol 17:897-908.

Lemasters JJ, Nieminen AL, Qian T, Trost LC, Elmore SP, Nishimura Y, Crowe RA, Cascio WE, Bradham CA, Brenner DA, Herman B (1998) The mitochondrial permeability transition in cell death: a common mechanism in necrosis, apoptosis and autophagy. Biochim Biophys Acta 1366:177-196.

Li M, Ona VO, Chen M, Kaul M, Tenneti L, Zhang X, Stieg PE, Lipton SA, Friedlander RM (2000) Functional role and therapeutic implications of neuronal caspase- 1 and -3 in a mouse model of traumatic spinal cord injury. Neuroscience 99:333-342.

Metivier D, Dallaporta B, Zamzami N, Larochette N, Susin SA, Marzo I, Kroemer G (1998) Cytofluorometric detection of mitochondrial alterations in early CD95/Fas/APO-1-triggered apoptosis of Jurkat T lymphoma cells. Comparison of seven mitochondrion-specific fluorochromes. Immunol Lett 61:157-163.

Mootha VK, Wei MC, Buttle KF, Scorrano L, Panoutsakopoulou V, Mannella CA, Korsmeyer SJ (2001) A reversible component of mitochondrial respiratory dysfunction in apoptosis can be rescued by exogenous cytochrome c. EMBO J 20:661-671.

Morris EJ, Geller HM (1996) Induction of neuronal apoptosis by camptothecin, an inhibitor of DNA topoisomerase-I: evidence for cell cycleindependent toxicity. J Cell Biol 134:757-770.

Morris EJ, Keramaris E, Rideout HJ, Slack RS, Dyson NJ, Stefanis L, Park DS (2001) Cyclin-dependent kinases and P53 pathways are activated independently and mediate Bax activation in neurons after DNA damage. J Neurosci 21:5017-5026.

Mortimore GE, Poso AR (1986) The lysosomal pathway of intracellular proteolysis in liver: regulation by amino acids. Adv Enzyme Regul 25:257-276.

Pampfer S, Cordi S, Vanderheyden I, Van Der Smissen P, Courtoy PJ, Van Cauwenberge A, Alexandre H, Donnay I, De Hertogh R (2001) Expression and role of $\mathrm{Bcl}-2$ in rat blastocysts exposed to high D-glucose. Diabetes 50:143-149.

Park DS, Morris EJ, Padmanabhan J, Shelanski ML, Geller HM, Greene LA (1998) Cyclin-dependent kinases participate in death of neurons evoked by DNA-damaging agents. J Cell Biol 143:457-467.

Petiot A, Ogier-Denis E, Blommaart EF, Meijer AJ, Codogno P (2000) Distinct classes of phosphatidylinositol $3^{\prime}$-kinases are involved in signaling pathways that control macroautophagy in HT-29 cells. J Biol Chem 275:992-998.

Radi R, Cassina A, Hodara R, Quijano C, Castro L (2002) Peroxynitrite reactions and formation in mitochondria. Free Radic Biol Med 33:1451-1464.

Ricci JE, Gottlieb RA, Green DR (2003) Caspase-mediated loss of mitochondrial function and generation of reactive oxygen species during apoptosis. J Cell Biol 160:65-75.

Rideout HJ, Stefanis L (2001) Caspase inhibition: a potential therapeutic strategy in neurological diseases. Histol Histopathol 16:895-908.

Rideout HJ, Stefanis L (2002) Proteasomal inhibition-induced inclusion formation and death in cortical neurons require transcription and ubiquitination. Mol Cell Neurosci 21:223-238.

Rideout HJ, Zang E, Yeasmin M, Gordon R, Jabado O, Park DS, Stefanis L (2001) Inhibitors of trypsin-like serine proteases prevent DNA damageinduced neuronal death by acting upstream of the mitochondrial checkpoint and of p53 induction. Neuroscience 107:339-352.

Rukenstein A, Rydel RE, Greene LA (1991) Multiple agents rescue PC12 cells from serum-free cell death by translation- and transcriptionindependent mechanisms. J Neurosci 11:2552-2563.

Saeki K, Yuo A, Okuma E, Yazaki Y, Susin SA, Kroemer G, Takaku F (2000) Bcl-2 down-regulation causes autophagy in a caspase-independent manner in human leukemic HL60 cells. Cell Death Differ 7:1263-1269.

Sakuma H, Yamamoto M, Okumura M, Kojima T, Maruyama T, Yasuda (2002) High glucose inhibits apoptosis in human coronary artery smooth muscle cells by increasing bcl-xL and bfl-1/A1. Am J Physiol Cell Physiol 283:C422-C428.

Schaffer SW, Croft CB, Solodushko V (2000) Cardioprotective effect of chronic hyperglycemia: effect on hypoxia-induced apoptosis and necrosis. Am J Physiol Heart Circ Physiol 278:H1948-H1954.

Schulz JB, Weller M, Moskowitz MA (1999) Caspases as treatment targets in stroke and neurodegenerative diseases. Ann Neurol 45:421-429.

Seglen PO, Gordon PB (1982) 3-Methyladenine: specific inhibitor of autophagic/lysosomal protein degradation in isolated rat hepatocytes. Proc Natl Acad Sci USA 79:1889-1892.

Selznick LA, Zheng TS, Flavell RA, Rakic P, Roth KA (2000) Amyloid betainduced neuronal death is bax-dependent but caspase-independent. J Neuropathol Exp Neurol 59:271-279.

Stefanis L, Troy CM, Qi H, Greene LA (1997) Inhibitors of trypsin-like serine proteases inhibit processing of the caspase Nedd-2 and protect PC12 cells and sympathetic neurons from death evoked by withdrawal of trophic support. J Neurochem 69:1425-1437. 
Stefanis L, Park DS, Friedman WJ, Greene LA (1999) Caspase-dependent and -independent death of camptothecin-treated embryonic cortical neurons. J Neurosci 19:6235-6247.

Stefanis L, Larsen KE, Rideout HJ, Sulzer D, Greene LA (2001) Expression of A53T mutant but not wild-type alpha-synuclein in PC12 cells induces alterations of the ubiquitin-dependent degradation system, loss of dopamine release, and autophagic cell death. J Neurosci 21:9549-9560.

Susin SA, Lorenzo HK, Zamzami N, Marzo I, Snow BE, Brothers GM, Mangion J, Jacotot E, Costantini P, Loeffler M, Larochette N, Goodlett DR, Aebersold R, Siderovski DP, Penninger JM, Kroemer G (1999) Molecular characterization of mitochondrial apoptosis-inducing factor. Nature 397:441-446.

Tennyson VM, Gershon MD, Sherman DL, Behringer RR, Raz R, Crotty DA, Wolgemuth DJ (1993) Structural abnormalities associated with congenital megacolon in transgenic mice that overexpress the Hoxa-4 gene. Dev Dyn 98:128-153.

Thiemermann C, McDonald MC, Cuzzocrea S (2001) The stable nitroxide, Tempol, attenuates the effects of peroxynitrite and oxygen-derived free radicals. Crit Care Med 29:223-224.

Troy CM, Rabacchi SA, Hohl JB, Angelastro JM, Greene LA, Shelanski ML (2001) Death in the balance: alternative participation of the caspase-2 and -9 pathways in neuronal death induced by nerve growth factor deprivation. J Neurosci 21:5007-5016.

van Deurs B, Holm PK, Sandvig K (1996) Inhibition of the vacuolar H(+)-
ATPase with bafilomycin reduces delivery of internalized molecules from mature multivesicular endosomes to lysosomes in HEp-2 cells. Eur J Cell Biol 69:343-350.

Wang X, Yang C, Chai J, Shi Y, Xue D (2002) Mechanisms of AIF-mediated apoptotic DNA degradation in Caenorhabditis elegans. Science 298:1587-1592.

Xiang H, Kinoshita Y, Knudson CM, Korsmeyer SJ, Schwartzkroin PA, Morrison RS (1998) Bax involvement in p53-mediated neuronal cell death. J Neurosci 18:1363-1373.

Xue L, Fletcher GC, Tolkovsky AM (1999) Autophagy is activated by apoptotic signalling in sympathetic neurons: an alternative mechanism of death execution. Mol Cell Neurosci 14:180-198.

Xue L, Fletcher GC, Tolkovsky AM (2001) Mitochondria are selectively eliminated from eukaryotic cells after blockade of caspases during apoptosis. Curr Biol 11:361-365.

Yamamoto A, Tagawa Y, Yoshimori T, Moriyama Y, Masaki R, Tashiro Y (1998) Bafilomycin Al prevents maturation of autophagic vacuoles by inhibiting fusion between autophagosomes and lysosomes in rat hepatoma cell line, H-4-II-E cells. Cell Struct Funct 23:33-42.

Zamzami N, Marchetti P, Castedo M, Decaudin D, Macho A, Hirsch T, Susin SA, Petit PX, Mignotte B, Kroemer G (1995) Sequential reduction of mitochondrial transmembrane potential and generation of reactive oxygen species in early programmed cell death. J Exp Med 182:367-377. 